\title{
Crescimento inicial de ipê branco (Handroanthus roseoalbus (Ridl.) Mattos) sob três intensidades luminosas
}

\author{
Initial growth of white ipê (Handroanthus roseoalbus (Ridl.) Mattos) under three light intensities \\ Crecimiento inicial de ipê blanco (Handroanthus roseoalbus (Ridl.) Mattos) bajo tres intensidades
} de luz

Reginaldo Almeida Andrade

ORCID: https://orcid.org/0000-0001-6547-4977 Universidade Federal do Acre, Brasil

E-mail: Reginaldo.andrade@ unir.br

Cleverson Agueiro de Carvalho

ORCID: https://orcid.org/0000-0002-3256-7023 Universidade Federal do Acre, Brasil

E-mail: Cleversoncarvalho92@gmail.com

Ítalo Felipe Nogueira Ribeiro

ORCID: https://orcid.org/0000-0003-0498-6949 Universidade Federal do Acre, Brasil E-mail: italo080@live.com

Rychaellen Silva de Brito

ORCID: https://orcid.org/0000-0002-3387-7218 Universidade Federal do Acre, Brasil E-mail: Rychaellenbrito@gmail.com

Rean Augusto Zaninetti

ORCID: https://orcid.org/0000-0002-3553-6556 Universidade Federal do Acre, Brasil E-mail: reanaugusto@gmail.com

Matheus Matos do Nascimento ORCID: https://orcid.org/0000-0003-0230-0042 Universidade Federal do Acre, Brasil E-mail: matheusxmattos@gmail.com

Davair Lopes Teixeira Junior ORCID: https://orcid.org/0000-0001-5051-9255 Universidade Federal do Acre, Brasil E-mail: davair.junior@ifac.edu.br

Regina Lúcia Félix Ferreira

ORCID: https://orcid.org/0000-0003-2401-4995 Universidade Federal do Acre, Brasil E-mail: Reginalff@yahoo.com.br

\begin{abstract}
Resumo
A produção de mudas em quantidade e qualidade é uma das etapas mais importantes no processo de implantação de povoamentos florestais. Para se obter sucesso nos viveiros, fatores como o tipo de substrato, volumes dos recipientes, tipo de fertilização, temperatura e luminosidade precisam ser conhecidos. O objetivo desse trabalho foi avaliar o desenvolvimento inicial de mudas de ipê-branco sob diferentes gradientes de luminosidade. O experimento foi realizado no município de Rio Branco, Acre, em um delineamento inteiramente casualizado, com três tratamentos e dez repetições. Os tratamentos foram a produção a pleno sol, com $50 \%$ e $70 \%$ de sombreamento. Foram avaliados a altura da planta, diâmetro do coleto, massa seca da parte aérea, massa seca da raiz, e massa seca total, além do índice de qualidade de Dickson. Constatou-se que as mudas de ipê-branco se desenvolvem adequadamente em ambiente pleno sol, onde são obtidas as mudas com os melhores indicadores de qualidade.
\end{abstract}

Palavras-chave: Espécies nativas; Fisiologia vegetal; Sombreamento; Viveiros florestais.

\begin{abstract}
The production of seedlings in quantity and quality is one of the most important steps in the process of implementing forest stands. To be successful in the nurseries, factors such as the type of substrate, container volumes, type of fertilization, temperature and luminosity need to be known. The objective of this work was to evaluate the initial development of white ipe seedlings under different light gradients. The experiment was carried out in the city of Rio Branco, Acre, in a completely randomized design, with three treatments and ten replications. The treatments were full sun production, with $50 \%$ and $70 \%$ shading. Plant height, stem diameter, shoot dry mass, root dry mass, and total dry
\end{abstract}


mass were evaluated, in addition to the Dickson quality index. It was found that the white ipe seedlings develop properly in full sun environment, where the seedlings with the best quality indicators are obtained.

Keywords: Native species; Plant physiology; Shading; Forest nurseries.

\section{Resumen}

Producción de plántulas en cantidad y calidad en una de las etapas más importantes del proceso de implantación de la planta floreciente. Para tener éxito en los viveros es necesario conocer factores como el tipo de sustrato, los volúmenes de los contenedores, el tipo de fertilización, la temperatura y la luminosidad. El objetivo de este trabajo fue evaluar el desarrollo inicial de plántulas de ipe blanco bajo diferentes gradientes de luminosidad. El experimento se realizó en la ciudad de Rio Branco, Acre, en un diseño completamente al azar, con tres tratamientos y diez repeticiones. Los tratamientos fueron de producción de pleno sol, con $50 \%$ y $70 \%$ de sombreado. Se evaluó la altura de la planta, el diámetro del tallo, la masa seca de los brotes, la masa seca de la raíz y la masa seca total, además del índice de calidad de Dickson. Se encontró que las plántulas de ipe blanco se desarrollan adecuadamente en ambiente de pleno sol, donde se obtienen las plántulas con los mejores indicadores de calidad.

Palabras clave: Especies nativas; Fisiología de las plantas; Sombreado; Viveros forestales.

\section{Introdução}

Espécies arbóreas nativas, como os ipês, apresentam uma inconfundível beleza ornamental, e mesmo com curtos períodos de florescimento, enobrecem a paisagem e agregam valor ecológico, além de serem frequentemente utilizadas em projetos de reflorestamento, de preservação ambiental e recuperação de áreas degradadas. Sua madeira apresenta alta densidade e elevada resistência mecânica, por isso é muito utilizada na construção civil e fabricação de móveis (Mezzomo et al., 2018; Berghetti et al., 2021).

Neste contexto, a espécie Handroanthus roseoalbus (Ridl.) Mattos, família Bignoneaceae, conhecida popularmente como ipê-branco, vem sendo amplamente explorada na região Amazônica, em planos de manejo florestal e também por meio da extração ilegal de madeira. Isto ocorre principalmente pela alta qualidade de sua madeira e potencial econômico que lhe é atribuído. Entretanto, como a exploração vem sendo realizada de forma intensa, o ipê é listado como espécie vulnerável e ameaçada de extinção (Bassegio et al., 2017).

Desta forma, é fundamental que se estabeleça novos povoamentos para que a restauração florestal ocorra, aliado a isso, adotar técnicas silviculturais que possam suprir essa demanda sem acarretar gastos onerosos. Neste contexto, o êxito de um povoamento florestal passa por critérios como formação de mudas de qualidades, e posteriormente, o desempenho à campo (Faria et al., 2019).

A luminosidade desempenha um papel importante em todos os estágios de desenvolvimento vegetal, podendo alterar diversos mecanismos fisiológicos da planta, e com isso, modificar o desenvolvimento dos diversos órgãos e tecidos (Taiz et al., 2017). Partindo do pressuposto que cada espécie responde de maneira distinta aos níveis de luminosidade que se é colocado, o sombreamento proporcionado em viveiros auxilia no desenvolvimento e posteriormente adaptação das mudas a campo (Maekawa et al., 2020).

Souza et al. (2019) afirmam que a depender de escolhas como recipientes, sombreamento e substrato a qualidade de mudas florestais pode ser comprometida, sendo, portanto, esses fatores os que devem ser observados e adequados para a espécie e assim se obter a melhor técnica de produção e manejo para que p tempo e gastos no viveiro sejam otimizados. O objetivo deste trabalho avaliar o efeito da luminosidade na produção de mudas de Handroanthus roseoalbus (Ridl.) Mattos.

\section{Metodologia}

O experimento foi realizado entre janeiro e maio de 2021 no viveiro florestal da Fundação de Tecnologia do Estado do Acre (FUNTAC), Rio Branco, AC. O delineamento estatístico foi inteiramente casualizado, com três tratamentos, constituídos pelos ambientes, pleno sol, $50 \%$ e $70 \%$ de sombreamento, com dez repetições. A presente pesquisa aborda 
aspectos experimentais qualitativos e foi estruturada em ambiente controlado, de forma que os dados produzidos possam ser analisados e validados por modelos estatísticos, garantindo confiabilidade nos resultados, conforme preconiza Pereira et al. (2018).

Foram utilizadas sementes coletadas em 2020 na microrregião de Rio Branco, no estado do Acre. Após a coleta, as sementes foram homogeneizadas para obtenção da fração sementes puras e a semeadura realizada em caixas de madeira $(1,5 \mathrm{x}$ $1,0 \times 0,2 \mathrm{~m}$ ) preenchidos com areia lavada e peneirada, irrigadas duas vezes ao dia durante 30 dias.

Trinta dias após a semeadura realizou a repicagem das plântulas normais, com tamanhos uniformes, para o substrato comercial, composto por casca de pinus bio-estabilizada, vermiculita, moinha de carvão vegetal e espuma fenólica, com adição de 6,0 kg m-3 de fertilizante de liberação controlada, Osmocote® na formulação NPK (15-10-10)

Aos 120 dias após a repicagem, as mudas foram retiradas do viveiro e levadas ao laboratório para realização da avaliação biométrica. As variáveis analisadas foram por meio da seleção ao acaso de dez mudas e as seguintes determinações:

a) DC: diâmetro do caule $(\mathrm{mm})$ - determinado na região do mesocótilo por meio de um paquímetro digital de precisão;

b) AP: altura da planta $(\mathrm{cm})$ - determinada com auxílio de régua graduada, tomando como referência a região compreendida entre o mesocótilo e o ponto de inserção do último par de folhas;

c) MSPA: massa seca da parte aérea (g) - determinada em balança de precisão 0,0001 g após secagem em estufa com circulação forçada de ar a uma temperatura constante de $70{ }^{\circ} \mathrm{C}$ até atingir massa constante;

d) MSR: massa seca da raiz $(\mathrm{g})$ - determinada em balança de precisão 0,0001 g após serem lavadas em água corrente e secas através da mesma metodologia da MSPA;

e) MST: massa seca total (g) - através do somatório da MSPA com a MSR,

f) IQD: Índice de Qualidade de Dickson (1960), usando a seguinte equação:

$$
\mathrm{IQD}=\frac{\operatorname{MST}(\mathrm{g})}{\left(\frac{\mathrm{A}(\mathrm{cm})}{\mathrm{DC}(\mathrm{mm})}+\frac{\operatorname{MSPA}(\mathrm{g})}{\operatorname{MSR}(\mathrm{g})}\right)}
$$

Os dados foram submetidos a detecção dados discrepantes, verificação das normalidades dos resíduos e homogeneidade de variâncias. Posteriormente foi efetuada a análise de variância dos dados e verificados pelo teste $\mathrm{F}$ a existência de diferença significativa $(\mathrm{p}<0,05)$ entre os tratamentos. As médias foram comparadas pelo teste de Tukey ao nível de 5\% de probabilidade com auxílio do software estatístico Sisvar.

\section{Resultados e Discussão}

O desenvolvimento vegetativo das mudas de ipê branco foi significativamente afetado pelos ambientes de cultivo (Tabela 1). As plantas cultivadas à pleno solo apresentaram os melhores indicadores de crescimento inicial, com maior altura de planta (AP), diâmetro do coleto (DC), massas secas da parte aérea (MSPA), das raízes (MSR) e total (MST), além do maior índice de qualidade de Dickson (IQD) (Tabela 2). 
Tabela 1. Resumo da análise de variância (ANOVA) para massas secas total (MST), parte aérea (MSPA), raiz (MSR) e índice de qualidade de Dickson (IQD).

\begin{tabular}{llcccccc}
\hline \multirow{2}{*}{ Fonte de variação } & \multirow{2}{*}{ GL } & AP & DC & \multicolumn{2}{c}{ MSPA } & MSR & MST \\
\cline { 3 - 7 } & & \multicolumn{5}{c}{ Quadrado médio } & \\
\hline Ambiente & 2 & $130,57^{*}$ & $4,10^{*}$ & $8,10^{*}$ & $9,06^{*}$ & $34,26^{*}$ & $1,82^{*}$ \\
Erro & 27 & 2,12 & 0,47 & 0,21 & 0,15 & 0,48 & 0,06 \\
\hline CV $(\%)$ & & 8,10 & 10,20 & 21,48 & 17,83 & 16,01 & 21,19 \\
\hline
\end{tabular}

CV: Coeficiente de variação; *significativo pelo Teste F a 5\% de probabilidade. Fonte: Autores (2021).

$\mathrm{O}$ crescimento em altura das mudas cultivadas à pleno sol foi $34 \%$ superiores àquelas produzidas no ambiente com $70 \%$ de sombreamento, indicando e que a alta irradiância favorece o desenvolvimento inicial de mudas de ipê branco. Resultados similares foram observados para as demais variáveis, com aumentos de 17,32\% no diâmetro do coleto; $246,3 \%$ na produção de massa seca da parte aérea; 249,6 na massa seca das raízes e 248\% da massa seca total (Tabela 2).

Tabela 2. Altura (A), diâmetro do coleto (DC), massas secas da parte aérea (MSPA), raiz (MSR) e total (MST), e índice de qualidade de Dickson (IQD) de plantas de ipê branco, aos 120 dias de idade, em função do sombreamento e volume de recipiente.

\begin{tabular}{lcccccc}
\hline \multirow{2}{*}{ Ambientes } & $\begin{array}{c}\text { AP } \\
\mathrm{cm}\end{array}$ & $\begin{array}{c}\text { DC } \\
\mathrm{mm}\end{array}$ & MSPA & MSR & MST & IQD \\
\cline { 4 - 6 } Pleno sol & $21,40 \mathrm{a}$ & $7,39 \mathrm{a}$ & $3,03 \mathrm{a}$ & $3,17 \mathrm{a}$ & $6,20 \mathrm{a}$ & $1,61 \mathrm{a}$ \\
$50 \%$ & $18,34 \mathrm{~b}$ & $6,79 \mathrm{ab}$ & $2,18 \mathrm{~b}$ & $2,18 \mathrm{~b}$ & $4,37 \mathrm{~b}$ & $1,18 \mathrm{~b}$ \\
$70 \%$ & $14,20 \mathrm{c}$ & $6,11 \mathrm{~b}$ & $1,23 \mathrm{c}$ & $1,27 \mathrm{c}$ & $2,50 \mathrm{c}$ & $0,76 \mathrm{c}$ \\
\hline
\end{tabular}

$\mathrm{CV}(\%)$

*Médias seguidas de letras iguais, na coluna, não diferem ( $\mathrm{p}>0,05)$ entre si pelo teste de Tukey. Fonte: Autores (2021).

As plantas conseguem absorver parte da radiação incidente do sol, e por uma série de mecanismos bioquímicos, utilizando CO2, água e nutrientes, convertem a luz em carboidratos através da fotossíntese (Kluge et al., 2015). Dentre os diversos fatores ambientais que influenciam as taxas de fotossíntese e respiração, a quantidade e intensidade luminosa é um dos mais importantes. A irradiância em que a taxa fotossintética líquida é igual a zero, ou seja, a troca de $\mathrm{CO} 2$ entre a folha e o ambiente é nula, é denominada de irradiância de compensação ou ponto de compensação luminoso. Nessa faixa de luminosidade, as plantas se mantêm em equilíbrio, não morrem, entretanto, o crescimento é nulo (Raven et al., 2013).

O ponto de compensação luminoso varia em função da espécie, da idade da planta, da temperatura, da concentração de $\mathrm{CO} 2$ na atmosfera, e da posição da folha na planta. Abaixo da irradiância de compensação ocorre perda líquida de $\mathrm{CO} 2$ através da respiração, podendo levar a planta à morte. Somente acima desse ponto se observa fotossíntese líquida e aumento na biomassa das plantas. Em plantas heliófilas como o ipê-branco, a irradiância de compensação encontra-se entre 10 a $20 \mu$ moles de fótons m-2 s-1. Por outro lado, nas plantas ombrófilas, ou de sombra, a irradiância de compensação varia entre 1 a $5 \mu$ moles de fótons m-2 s-1, o que pode ser atribuído a sua baixa taxa respiratória, permitindo um ganho líquido de carbono mesmo em ambientes limitados por luz (Raven et al., 2013).

Em espécies heliófilas, como os ipês, que são intolerantes a ambientes com elevado sombreamento, por possuir alto ponto de compensação luminoso, a alta irradiância altera a concentração de proteínas nas folhas, gerando o acúmulo de estruturas fotossintética, resultando em maior produção de carboidratos por unidade de área foliar, deste modo, quando a disponibilidade de luz é abundante, o crescimento da planta aumenta (Guerra et al., 2015; Logendre \& Iersel, 2021).

Além disso, sob condições de baixa luminosidade, ocorre redução na produção de massa seca, pois, sob intensidade de 
luz abaixo do ponto de compensação o hidrato de carbono é mais consumido pela respiração do que produzido pela fotossíntese (Ortega et al., 2006). Além disso, as espécies heliófilas alteram a partição de fotoassimilados entre as diversas partes da planta, destinado mais reservas para o crescimento da área foliar, a fim de promover a máxima eficiência na captura de luz. Mudanças estruturais e fisiológicas promovidas em ambientes com pouca luminosidade aumentam a eficiência de captura de luz, mas reduzem o potencial fotossintético e vice-versa (Falcioni et al., 2018).

$\mathrm{O}$ aumento na produção de massa da matéria seca da parte aérea, raiz e total das mudas de ipê branco produzidas a pleno sol demonstra que a espécie é amplamente adaptada para crescer e se desenvolver sob condições adversas do campo (Oliveira \& Perez, 2012).

O desenvolvimento do sistema radicular é favorecido em ambiente pleno sol, com produção de 2,5 vezes maior do que as plantas em $70 \%$ de sombreamento. Este comportamento pode ser explicado como resposta estratégica da planta para superar épocas desfavoráveis, visto que as condições termo hídrica são estressantes, com isso ocorre o acumulo de reserva na parte radicular, resultado de maior concentração de fotoassimilados (Sousa et al., 2019).

Verificou-se que o índice de qualidade de Dickson (IQD) foi influenciado pelos ambientes de produção, com aumento da qualidade das mudas produzidas a pleno sol. Nessa condição, o IQD foi $211 \%$ acima do ambiente com $70 \%$ de restrição luminosa e $136 \%$ acima do tratamento com $50 \%$ de sombreamento. O índice de qualidade de Dickson é considerado um indicador ideal para expressar a qualidade de mudas, pois agrega variáveis morfológicas na equação (Sousa et al., 2019). Mota (2013), ao trabalharem com angico (Anadenanthera falcata), constataram que o tratamento sem interceptação luminosa apresentou maior IQD.

Observando as correlações de Pearson verifica-se que todos os parâmetros de crescimento das mudas de ipê branco são positivamente correlacionados, com coeficientes acima de 0,63 (Figura 1).

Figura 1.

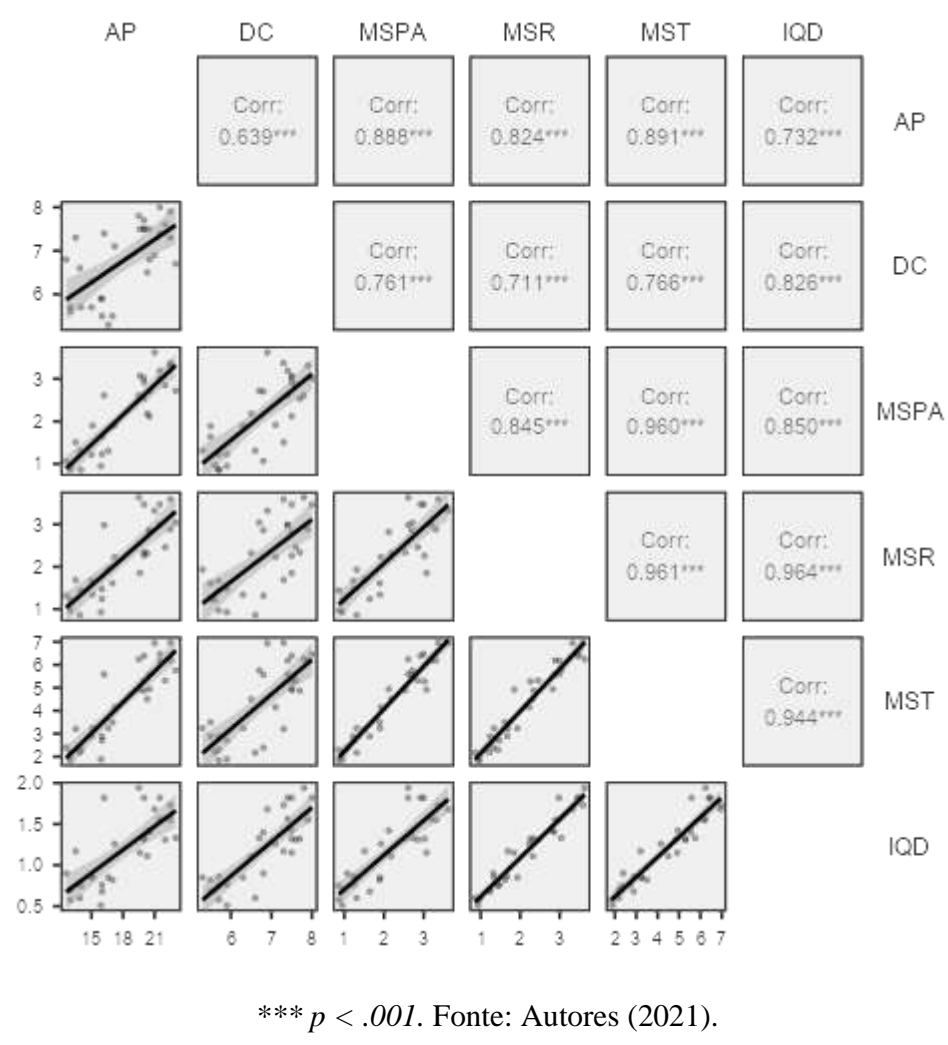


Nota-se que a altura da planta tem correlação positiva forte com a massa seca da parte aérea, com a massa seca das raízes e com o índice de qualidade e de Dickson, (0,88, 0,82 e 0,73 respectivamente), indicando crescimento equilibrado entre as partes da planta. Ou seja, à medida que ocorre desenvolvimento em altura, ocorre aumento do desenvolvimento de raízes, garantindo robustez à muda e maior capacidade de sobrevivência à campo.

Da mesma forma, o diâmetro do coleto demonstrou-se fortemente correlacionado com o IQD $(0,85)$. O diâmetro do coleto, assim como o IQD, é um forte indicador de qualidade, e para florestais, está diretamente associado ao desenvolvimento da parte aérea e sistema radicular, o que favorece a sobrevivência e o desenvolvimento das mudas após o plantio (Grave et al., 2007).

\section{Conclusão}

Mudas a pleno sol apresentam melhor crescimento e qualidade até os 120 dias de idade.

\section{Referências}

Bassegio, C., Fogaça, L. A., Baltazar, P. \& Emmel, E. (2017). Desenvolvimento de ipê-roxo em meios de cultura e concentrações de bap (6-benzilaminopurna) durante a etapa de multiplicação in vitro. Acta Iguazu, 6 (1), 72-80.

Berghetti, Á. L. P., Araujo, M. M., Tabaldi, L. A., Turchetto, F., Tassinari, A., Bernardy, D., Griebeler, A. M., Barbosa, F. M., Aimi, S. C. \& Brunetto, G. (2021). Effects of Nitrogen Fertilization on the Growth and on Photochemical Efficiency in Plants of Handroanthus heptaphyllus. Journal of Plant Nutrition, 44 (16), 2464-2475.

Dickson, A., Leaf, A. L. \& Hosner, J. F. (1960). Quality appraisal of white spruce and white pine seedling stock in nurseries. Forestry Chronicle, 36 (1), 1013 .

Falcioni, R., Moriwaki, T., Benedito, E., Bonato, C. M., Souza, L. A. \& Antunes, W. C. (2018). Increased gibberellin levels enhance light capture efficiency in tobacco plants and promote dry matter accumulation. Theoretical and Experimental Plant Physiology, 30 (1), $235-250$.

Faria, J. C. T., Lopes, D. A., Lyra, G. B., Melo, H. C. \& Melo, L. A. (2019). Manejo da densidade de plantas durante a produção de mudas em viveiro. Ciência Florestal, 29 (3), 1187-1198.

Grave, F., Franco, E. T. H., Pacheco, J. P. \& Santos, S. R. (2007). Crescimento de plantas jovens de Açoita-cavalo em quatro diferentes substratos. Ciência Florestal, 17 (4), 289-298.

Guerra, A., Santos, L. S., Gonçalves, L. G. \& Medri, C. (2015). Morfoanatomia de folhas de sol e de sombra de Handroanthus chrysotrichus (Mart. Ex DC.) Mattos (Bignoniaceae). Revista de Saúde e Biologia, 10 (1), 59-71.

Kluge, R. A., Tezotto-Uliana, J. V. \& Silva, P. P. M. (2015). Aspectos fisiológicos e ambientais da fotossíntese. Revista Virtual Química, 7 (1), $56-73$.

Logendre, R. \& Iersel, M. W. V. (2021). Supplemental far-red light stimulates lettuce growth disentangling morphological and physiological effects. Plants, $10(166), 1-21$.

Maekawa, L., Coelho, M. F. B. \& Weber, O. L. S. (2020). Substratos e restrição luminosa na produção de mudas de Ficus gomelleira Kunth. Revista Ciência Agraria, 63, 1-7.

Mezzomo, J. C., Araujo, M. M., Turchetto, F., Rorato, D. G., Griebeler, A. M., Berghetti, Á. L. P. \& Barbosa, F. M. (2018). Silvicultural Potential of Handroanthus heptaphyllus Under Doses of Controlled Release Fertilizer and Container Volume, in Nursery and in the Field. Journal of Agricultural Science, $10(11), 389-40$

Mota, L. H. S., Scalon, S. P. Q. \& Mussury, R. M. (2013). Efeito do condicionamento osmótico e sombreamento na germinação e no crescimento inicial das mudas de angico (Anadenathera falcata Benth. Speg.). Revista Brasileira de Plantas Medicinais, 15 (4), $655-663$.

Oliveira, A. K. M. \& Perez, S. C. J. G. A. (2012). Crescimento inicial de Tabebuia aurea sob três intensidade luminosas. Ciência Florestal, 22 (2), $263-273$.

Ortega, A. R., Almeida, L. S., Maia, N. \& Angelo A. C. (2006). Avaliação do crescimento de mudas de Psidium cattleianum Sabine a diferentes níveis de sombreamento em viveiro. Cerne, 12 (3), 300-308.

Pereira, A. S., Shitsuka, D. M., Parreira, F. J., \& Shitsuka, R. (2018). Metodologia da Pesquisa Cientifica. UFSM.

Raven, P. H., Evert, R. F. \& Eichhorn, S. E. (2013). Biologia Vegetal, 7a. ed. Rio de Janeiro: Guanabara Koogan.

Sousa, H. G. A., Duarte, V. B. R., Borges, A. V. S. \& Souza, P. B. (2019). Ambientes na emergência e desenvolvimento de Tabebuia serratifolia (Vahl) G. Nichols. Scientia Agraria Paranaensis, 18 (3), 276-281.

Souza, J. C., Pedrozo, C. A., Silva, K., Oliveira, V. X. A. \& Alencar, A. M. S. (2019). Ambientes para a produção de mudas e nodulação por rizóbios em Tachigali vulgaris. Ciência Florestal, 29 (1), 116-129.

Taiz, L., Zeiger, E., Moller, I. M. \& Murphy, A. (2017). Fisiologia e desenvolvimento vegetal. (6a ed.), Artmed. 